\title{
Prostatectomía radical asistida por robot da Vinci: experiencia inicial en 50 casos consecutivos*
}

\author{
Drs. OCTAVIO A. CASTILLO C..$^{1,2,3}$, GASTÓN LÓPEZ-FONTANA ${ }^{1}$, \\ ARQUÍMEDES RODRÍGUEZ-CARLIN ${ }^{1}$, EDUARDO LANDERER L. ${ }^{1,2}$, \\ IVAR VIDAL-MORA. ${ }^{1}$; MARÍA A. DE ORÚE-RÍOS²; REYNALDO GÓMEZ I. ${ }^{1}$ \\ 1 Departamento de Urología y Centro de Cirugía Robótica, Clínica Indisa. \\ 2 Facultad de Medicina, Universidad Andrés Bello. \\ 3 Facultad de Medicina, Universidad de Chile. \\ Santiago, Chile.
}

Abstract

\section{Da Vinci robot-assisted radical prostatectomy: initial experience in 50 consecutive cases}

Objective: To report our initial experience in 50 cases submitted to a Robotic Radical Prostatectomy (RRP), evaluating results and the learning curve. Material and Methods: From january to october 2010 we performed 50 consecutives cases of RRP with the da Vinci S-HD Surgical System ${ }^{\circledR}$. The database was performed prospectively, and was analyzed retrospectively. We evaluate demographic data (age, body mass index) and perioperative data such as clinical stage, preoperative PSA (Prostate Specific Antigen), Gleason Score, ASA, operative times, estimated blood loss, morbidity, hospital stay, time of bladder catheterization and positive margins. A statistical analysis of exponential regression was performed to estimate the learning curve. Results: The mean age was 62 years and the most frequent clinical stage was T1c (84\%). The mean PSA was $6.36 \mathrm{ng} / \mathrm{mL}$ and in $50 \%$ of the patients the Gleason Score was 7. The median surgical time was 199 minutes. The mean blood loss was $666 \mathrm{~mL}(50-4.000 \mathrm{~mL})$. The hospital stay and the average bladder catheterization time were 2 and 6 days, respectively. There were 2 conversions to a laparoscopic approach, none to open surgery, and $8 \%$ of postoperative complication (all Clavien 1). Inmediat urinary continence and potency rates were $88.3 \%$ and $33.3 \%$, respectively. When comparing the 25 initial cases versus the last 25 , there was a decrease in surgical time and estimated blood loss (254 minutes vs 189 minutes and $876 \mathrm{~mL}$ vs $467 \mathrm{~mL}$, respectively). We also found a lower rate of positive margins ( $20 \%$ vs $12 \%$ ). The learning curve statistically estimated is 40 procedures. Conclusion: The surgeon's experience determine a decrease in surgical time, intraoperative bleeding and especially in the rate of positive margins.

Key words: Prostatic neoplasms, prostate cancer, radical prostatectomy, robotic surgery.

*Recibido el 23 de febrero de 2011 y aceptado para publicación el 14 de junio de 2011.

No existe ningún interés financiero o comercial en este manuscrito.

Correspondencia: Dr. Octavio A. Castillo C.

Apoquindo 3990, Of. 809, Santiago, Chile.

C.P.: 7550112 - Fax (56-2) 2282425

octavio.castillo@indisa.cl 


\section{Resumen}

Objetivo: Comunicar nuestra experiencia inicial en 50 casos de Prostatectomía Radical Robótica (PRR), evaluando resultados y curva de aprendizaje. Material y Métodos: Desde enero a octubre de 2010 se realizaron 50 PRR con el sistema da Vinci S-HD ${ }^{\circledR}$. La base de datos fue confeccionada en forma prospectiva y se evaluaron en forma retrospectiva los datos demográficos (edad, índice de masa corporal), estadio clínico, valor de Antígeno Prostático Específico (APE), Score de Gleason, ASA, tiempos quirúrgicos, sangrado estimado, complicaciones, estadía hospitalaria, tiempo de sonda vesical y tasa de márgenes positivos. Se realizó un análisis estadístico de regresión exponencial para estimar la curva de aprendizaje del método. Resultados: La edad media fue de 62 años y el estadio clínico más frecuente fue el T1c (84\%). El valor medio de APE fue de 6,36 ng/mL. El score de Gleason en un 50\% correspondió al 7 y la media del ASA a 2. La mediana del tiempo quirúrgico fue de 199 minutos. El sangrado medio estimado fue de $666 \mathrm{~mL}$ (50-4.000 mL). La media de la estadía hospitalaria y el tiempo de sonda fueron de 2 y 6 días, respectivamente. Hubo 2 conversiones a cirugía laparoscópica, ninguna a cirugía abierta y un 8\% de complicaciones postoperatorias (todas Clavien 1). La tasa de continencia y de potencia inmediata fue de $88,3 \%$ y 33,3\%, respectivamente. Cuando comparamos los 25 casos iniciales versus los 25 finales hubo un descenso significativo en el tiempo quirúrgico y sangrado estimado (254 minutos vs 189 minutos y 876 mL vs 467 mL, respectivamente). También encontramos una menor tasa de márgenes positivos en el grupo 2 (12\% vs 20\%). El análisis estadístico determinó la curva de aprendizaje en 40 procedimientos. Conclusión: Una mayor experiencia del cirujano, determina una disminución en los tiempos quirúrgicos, sangrado intraoperatorio y sobre todo en la tasa de márgenes positivos.

Palabras clave: Neoplasias prostáticas, cáncer de próstata, prostatectomía radical, cirugía robótica.

\section{Introducción}

La incidencia de cáncer de próstata (CaP) en la actualidad es de aproximadamente 186.000 casos nuevos diagnosticados anualmente en los Estados Unidos $^{1}$ y su diagnóstico ha aumentado en forma considerable, tal es así que actualmente representa casi el 33\% de todos los cánceres diagnosticados en hombres $^{2}$. En nuestro país, la prevalencia publicada corresponde al $6,4 \%^{3}$, sin embargo, la incidencia es desconocida, estimándose una tasa de entre 55 y 57 por 100.000 habitantes en los últimos años ${ }^{4}$. Debido a que aproximadamente el $90 \%$ de los CaP son localizados, el tratamiento quirúrgico continúa siendo el tratamiento más común y el único que ha demostrado una ventaja en la sobrevida en comparación con la observación ${ }^{5}$.

Desde el primer caso de prostatectomía radical robótica realizado en el año 2000 por Binder y Kramer en Alemania ${ }^{6}$, con la utilización del sistema da Vinci (Intuitive Surgical, Inc., Sunnyvale, CA, USA), se ha incrementado su uso a nivel mundial, estimándose que el 66\% (2/3) de las prostatectomías radicales en el año 2008 fueron asistidas por robot en los Estados Unidos ${ }^{7}$. Dicha difusión aparentemente es debida a que la transición de cirugía abierta a robótica es más rápida que a cirugía laparoscópica y a las potenciales ventajas que ofrece la asistencia robótica: una lente binocular que permite la visualización del campo quirúrgico en 3 dimensiones (3-D) con un aumento de 10x, el movimiento de sus brazos articulados y la ausencia del temblor, lo que permitiría una mayor precisión de los movimientos, sin dejar de mencionar la mayor ergonomía que le proporciona al cirujano.

El objetivo de este trabajo es presentar nuestra experiencia inicial en 50 casos consecutivos de PRR asistida por el sistema da Vinci S-HD.

\section{Material y Método}

Desde la incorporación del robot da Vinci S-HD a nuestra Unidad, se han realizado 50 PRR consecutivas entre enero y octubre de 2010, por un mismo cirujano (OAC). En todos los casos la vía de abordaje fue la transperitoneal utilizando la técnica descrita por Patel $^{8}$. El paciente es colocado en posición de Trendelemburg, en un ángulo de $25^{\circ}$ con sujetador de hombros, cuidando todos los puntos de apoyo. Los puertos robóticos se posicionan como lo indica la Figura 1 y el robot es colocado entre las piernas del paciente como lo muestra la Figura 2.

La base de datos fue confeccionada en forma prospectiva y evaluada en forma retrospectiva. Los datos analizados fueron: edad, índice de masa corporal (IMC), estadio clínico, valor de antígeno prostático específico (APE) preoperatorio, Score de Gleason, presencia de cirugías previas, enfermedades asociadas, ASA (Riesgo según la Sociedad Americana de Anestesiología), tiempo quirúrgico (evaluando por separado el tiempo de colocación de trócares, de montaje y el tiempo de consola), preservación de las bandeletas neurovasculares, sangrado intraoperatorio estimado, complicaciones intra y postoperatorias, estadía hospitalaria, tiempo de sonda vesical y tasa de márgenes positivos. 


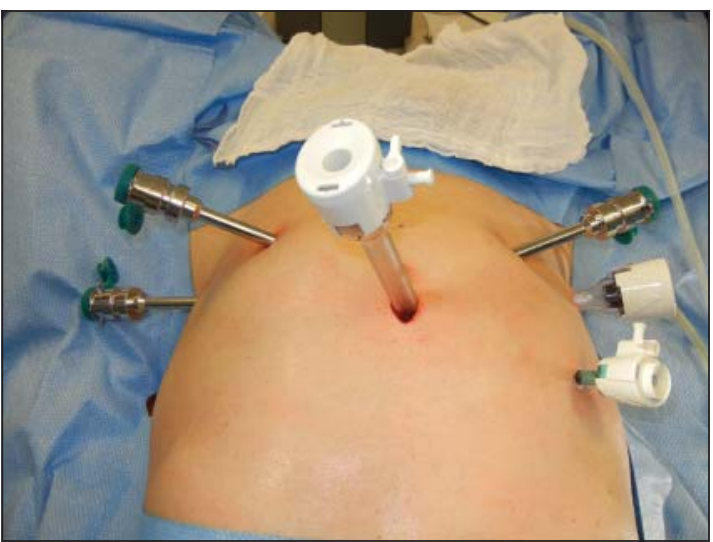

Figura 1. Colocación de puertos para prostatectomía radical robótica. Puerto de 12 milímetros supraumbilical (cámara), puertos robóticos de 8 milímetros infraumbilical y paramedial (brazo 1 y 2), puerto robótico de 8 milímetros a 3 centímetros por encima de la espina ilíaca anterosuperior izquierda (brazo 3). Un trocar de 10 milímetros a 3 centímetros por encima de la espina ilíaca anterosuperior derecha (ayudante) y uno de 5 milímetros a nivel subcostal derecho.

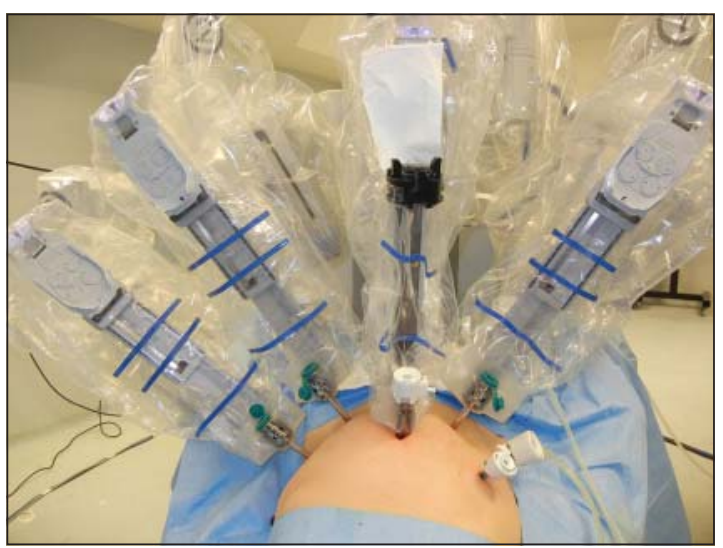

Figura 2. Se aprecia el robot montado sobre los puertos ingresando entre las piernas del paciente.

A pesar del corto período de seguimiento, evaluamos los resultados oncológicos y también los funcionales, continencia urinaria y función eréctil (Trifecta). Definimos un paciente continente a aquel que en el postoperatorio no requiere del uso de paños absorbentes y no presenta escape de orina con los esfuerzos. Para objeto del análisis la dividimos en 3 períodos: continencia inmediata al retiro de la sonda vesical, a los 3 y 6 meses de seguimiento. Para evaluar la potencia sexual, se utilizó el cuestionario de función eréctil o IIEF (International Index of Erectile Function Questionnaire) realizado previo a la cirugía y luego a 1,3 y 6 meses $^{10}$. Definimos como potencia conservada a aquel paciente capaz de penetrar a su pareja con o sin uso de drogas y un valor de IIEF superior a 25. Los resultados oncológicos fueron evaluados mediante la presencia de márgenes positivos en la biopsia quirúrgica definitiva y la recidiva bioquímica en el seguimiento.

Con el propósito de determinar la curva de aprendizaje, dividimos la serie en 2 grupos de 25 casos. Para cada uno de ellos evaluamos el tiempo quirúrgico (suma de los tiempos de trócares, montaje y consola), sangrado estimado y tasa de márgenes positivos. Para el análisis estadístico se ajustó un modelo de regresión exponencial, previa evaluación de la información, de la forma $Y=b_{1} * b_{2}^{\text {(intervención), }}$ lo que permite establecer la curva de aprendizaje, considerando a $b_{1}$ como la media del procedimiento y $b_{2}$ como la tasa de aprendizaje por cada intervención realizada. Un dato de interés en los análisis de curva de aprendizaje es la cantidad de procedimientos necesarios para reducir el valor inicial a la mitad.

\section{Resultados}

La edad media fue de 62 años (47 a 77) y el IMC de 27 (20 a 32,1). El estadio clínico más frecuente fue el T1c en un 84\% (42/50) y el resto fue T2a en $14 \%$ y un paciente con estadio T2c (2\%). La media de PSA preoperatorio correspondió a un 6,36 ng/ mL $(2,24-19,2)$ y el Score de Gleason más frecuente fue el 7 en un 50\% (25/50). Trece pacientes (26\%) tenían el antecedente de cirugía abdominal previa lo cual no dificultó la colocación de trócares. Treinta y siete (74\%) pacientes tenían enfermedades asociadas siendo la más frecuente la hipertensión arterial en un $64,8 \%$ (24/37), y la media del puntaje del ASA fue 2 (1 a 3). En el mismo acto operatorio, a un paciente (número 36 de la serie) se le efectuó además una hernioplastía inguinal derecha con malla.

Los tiempos quirúrgicos de la cirugía robótica se dividen en 3: el tiempo de trócares, que consiste en el tiempo que demora la colocación de los puertos; el tiempo de montaje del robot en los puertos y finalmente el tiempo de consola que es el tiempo que trascurre desde el inicio hasta el final del manejo robótico por parte del cirujano. La media del tiempo de trócares, montaje y consola fue de 15 minutos (7 a 60 minutos), 9 minutos (3 a 20 minutos) y 201 minutos (95 a 480), respectivamente. La media del tiempo quirúrgico definido como la suma de los tres tiempos fue de 221,7 minutos (110 a 500 minutos). A 8 pacientes (16\%) se les efectuó una linfadenectomía ilioobturatriz extendida. La reconstrucción del rabdoesfínter, según la técnica de Rocco, fue efectuada en todos los pacientes ${ }^{9}$. Las bandeletas 
neurovasculares fueron conservadas en el $82 \%$ de los casos.

En cuanto al sangrado intraoperatorio estimado, la media fue de $676 \mathrm{~mL}$ con un rango de 50 a 4.000 $\mathrm{mL}$. La tasa de transfusión fue del 6\% (3/50), dos de ellos fueron nuestros primeros 2 casos y el otro correspondió al número 47 que requirió una unidad de glóbulos rojos previo a la cirugía debido a una cardiopatía isquémica con anemia secundaria. Sólo 2 pacientes (4\%) fueron convertidos a cirugía laparoscópica por dificultad en la confección de la anastomosis probablemente debido a una incompleta liberación de la vejiga (los 2 primeros pacientes de la serie). Hubo cuatro pacientes (8\%) con complicaciones postoperatorias. Éstas fueron una fístula urinaria que resolvió espontáneamente con tratamiento conservador, un paciente evolucionó con íleo adinámico, y 2 presentaron una retención aguda de orina que se manejó con sonda vesical por 72 hrs. Todas estas complicaciones fueron Clavien $1^{11}$. La estadía hospitalaria media fue de 2 días ( 2 a 6 días) y el tiempo de sonda vesical fue de 6 días (4 a 10 días). Finalmente, la tasa global de márgenes positivos fue del 18\% (9/50).

Si bien el período de seguimiento es corto, siendo la media de 8,34 meses (3 a 12 meses), analizamos los resultados iniciales en cuanto a la continencia urinaria, función sexual y resultados oncológicos (Trifecta). De un total de 49 pacientes evaluados, con seguimiento hasta 6 meses, el porcentaje de continencia inmediata al retiro de sonda fue de $77,5 \%$ (38 pacientes), elevándose a 85,7\% (42 pacientes) a los 3 meses, y de $87,7 \%$ (43 pacientes) a los 6 meses. El resto de los pacientes presenta incontinencia leve que los obliga a usar 1 protector al día por escape con esfuerzos.
Según los criterios previamente descritos relacionado con la potencia sexual, en 39 pacientes evaluables en quienes se hizo preservación de bandeletas neurovasculares y con IIEF igual o superior a 25 , el porcentaje de pacientes con potencia inmediata al $1^{\circ}$ mes fue de $33,33 \%$ (13 pacientes), valor que se elevó a 53,8\% (21 pacientes) a los 3 meses y de $56,4 \%$ (22 pacientes) a los 6 meses de seguimiento. Es importante destacar que en términos de continencia y potencia, los resultados definitivos debieran ser evaluados después de 12 meses de la cirugía.

A 8,34 meses de seguimiento promedio, tenemos sólo dos pacientes (4\%) con recidiva bioquímica, ambos con margen quirúrgico positivo y derivado a radioterapia complementaria.

La comparación de la serie dividida en 2 grupos de 25 pacientes se resume en la Tabla 1. El tiempo quirúrgico promedio correspondiente a la suma de los tres (trócares, montaje y consola) disminuyó de 254 a 189 minutos. El sangrado intraoperatorio estimado para el grupo 1 fue de 876 mL (200-4.000 $\mathrm{mL})$ y para el grupo 2 de $467 \mathrm{~mL}(50-1.000 \mathrm{~mL})$. La tasa de márgenes quirúrgicos positivos fue de 20 y $12 \%$, respectivamente.

Ambas curvas de aprendizajes son significativas al modelo exponencial, alcanzando ajustes de $91 \%$ y $60 \%$ para las variables tiempo quirúrgico y sangrado intraoperatorio, respectivamente (Tabla 2). La información observada y estimada por el modelo de regresión exponencial es presentada en las Figuras 3 y 4 para las variables tiempo quirúrgico y sangrado intraoperatorio, respectivamente. Estos gráficos demuestran los parámetros de tiempo de consola y sangrado intraoperatorio relacionados con el número de casos operados, apreciándose una pendiente negativa en la curva a mayor experiencia del cirujano.

Tabla 1. Datos de la serie actual divididos en 2 grupos de 25 cada uno

\begin{tabular}{|lcc|}
\hline Parámetros & 25 iniciales (Grupo 1) & 25 finales (Grupo 2) \\
\hline Edad & $62(46-74)$ & 61 (47-74) \\
Estudio clínico & T1c (80\%) & T1c (88\%) \\
& T2a (15\%) (12\%) \\
Tiempo quirúrgico promedio (minutos) & T2c (4\%) & 189 \\
Sangrado intraoperatorio estimado promedio (mL) & 254 & 467 \\
Tasa de transfusión & 876 & $4 \%(1 / 25)$ \\
Tasa de complicaciones postoperatorio & $8 \%(2 / 25)$ & $8 \%(2 / 25)$ \\
& $8 \%(2 / 25)$ & Clavien I 0\% \\
Tasa de conversión & Clavien I 0\% & Clavien II a V \\
Tasa de márgenes positivos & Clavien II a V & $0 \%$ \\
\hline
\end{tabular}


PROSTATECTOMÍA RADICAL ASISTIDA POR ROBOT DA VINCI: EXPERIENCIA INICIAL EN 50 CASOS ...

Tabla 2. Modelos de regresión exponencial para las variables tiempo y sangrado

\begin{tabular}{|lccccrc|}
\hline Variables & Valor & SE & t & p-valor & LCI & UCI \\
Tiempo & & & & & \\
b1 & 295,81 & 22,65 & 13,06 & 0,000 & 250,27 & 341,34 \\
b2 & 0,9835 & 0,0032 & 302,99 & 0,000 & 0,9770 & 0,9900 \\
R $_{2}$ & 0,913 & & & & & 1825,99 \\
RMSE & 64,48257 & & & & & 0,9889 \\
Sangrado & & & 5,35 & 0,0000 & 827,86 & 0,9490 \\
b1 & 1326,92 & 248,21 & 97,78 & 0,0000 & & \\
b2 & 0,9690 & 0,0099 & & & & \\
R $_{2}$ & 0,5963 & & & & & \\
RMSE & 604,32 & & & & & \\
\hline
\end{tabular}

SE: Error estándar. t: Valor de t calculado para el coeficiente. p-valor: Significancia del coeficiente en el modelo. LCI: Límite inferior del intervalo de confianza al 95\% del coeficiente. UCI: Límite superior del intervalo de confianza al 95\% del coeficiente. R2: Coeficiente de determinación del modelo. RMSE: Error en la estimación del modelo.

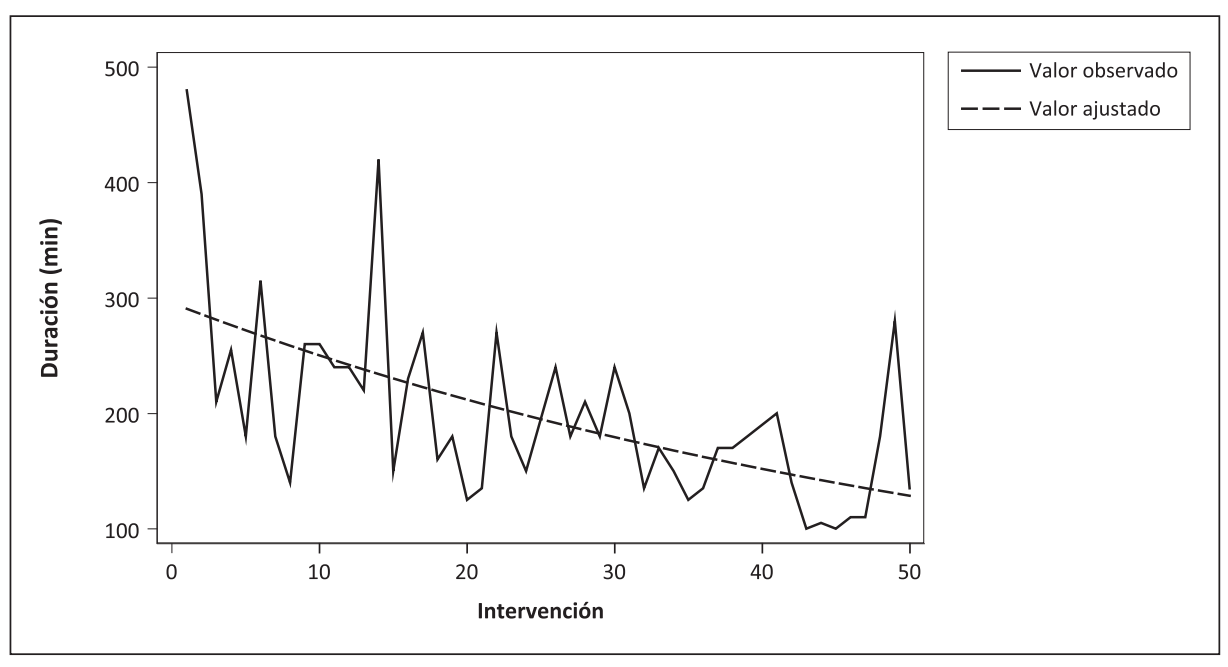

Figura 3. Valores ajustado por el modelo de regresión exponencial (línea discontinua) para la variable tiempo.

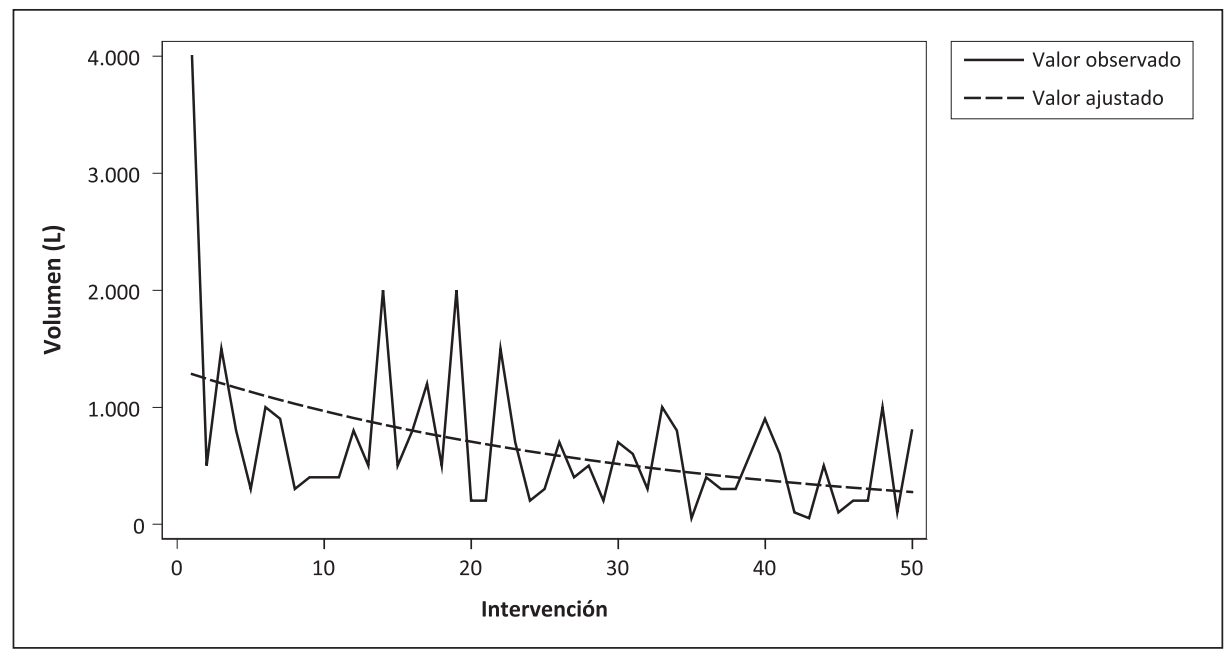

Figura 4. Valores ajustado por el modelo de regresión exponencial (línea discontinua) para la variable sangrado.

Rev. Chilena de Cirugía. Vol 63 - No 6, Diciembre 2011; pág. 609-616 
Los modelos de regresión estiman que los valores iniciales de ambas variables para este procedimiento alcanzan los 300 minutos y los $1.300 \mathrm{~mL}$, que las tasas de aprendizaje son de un $2 \%$ y $3 \%$, respectivamente, es decir, por cada procedimiento que el cirujano realiza, demora como media un $2 \%$ menos y presenta un 3\% menos de sangrado. Demostramos estadísticamente que sería necesario más de 40 procedimientos para lograr disminuir a la mitad los valores iniciales en ambas variables.

\section{Discusión}

Desde el inicio de la cirugía robótica con el sistema daVinci en 1999, y su posterior aprobación por la FDA (Food and Drug Administration) al año siguiente, su utilización ha ido aumentando, de tal forma que en la actualidad más del $60 \%$ de las PR en los Estados Unidos son abordadas en forma robótica $^{7}$. Además, cumpliendo 10 años de la primera PRR, han comenzado a comunicarse resultados a largo plazo relacionados a la Trifecta (continencia urinaria, función sexual y resultados oncológicos) con resultados similares y, en algunas series, superiores a la cirugía abierta ${ }^{12-14}$.

Los parámetros perioperatorios evaluados habitualmente en distintas series son el tiempo operatorio, sangrado estimado, tasa de transfusión, estadía hospitalaria y complicaciones perioperatorias. En referencia a esto, Coelho y cols, publicaron recientemente los resultados de la PRR en una revisión exhaustiva de la literatura ${ }^{15}$. En este trabajo el tiempo operatorio medio fue de 166,6 minutos con un rango entre 105 y 236 minutos. Patel y cols, comunican su experiencia en 1.500 PRR, siendo el tiempo medio de cirugía de 105 minutos $^{12}$. También comparan los primeros 300 casos vs los segundos 300 casos, encontrando un descenso en el tiempo operatorio de 120 a 105 minutos. En sus últimas 500 PRR el tiempo medio fue de 78 minutos. Badani y cols, publicaron también su experiencia en 2.766 casos $^{14}$. El tiempo operatorio promedio calculado desde la colocación de la aguja de Veress hasta el cierre de la piel fue de 154 minutos. Compararon los primeros 200 casos con sus últimos 200, siendo el tiempo quirúrgico medio de 160 y 131 minutos respectivamente. En nuestra serie inicial el tiempo promedio de cirugía fue de 221,7 minutos con un importante descenso en el grupo 2 (254 minutos para el grupo 1 y 189 minutos para el grupo 2).

En relación al sangrado intraoperatorio estimado, la media reportada por Coelho y cols, fue de $169 \mathrm{~mL}$ (103-609 mL), y la tasa de transfusión de 1,39\% (0,5 a $3 \%)^{15}$. La estadía hospitalaria varía entre 1 y 5,4 días, con un promedio de 1,55 días. La tasa media de complicaciones reportada para la PRR fue de 10,5\% pero al no existir una clasificación estándar sobre las complicaciones entre distintas publicaciones, es un parámetro difícil de evaluar. Sin embargo, desde la utilización de la clasificación de Clavien con su última modificación, diferentes reportes han publicado sus tasas de complicaciones ${ }^{11}$. Badani y cols, en su serie de 2.766 casos, reportaron una tasa del 12,2\% (Clavien I, 8\%; Clavien II, 3,7\%; Clavien III, 13\%; Clavien IV, 0,01\%; Clavien V, $<0,01 \%)^{14}$. En cambio, Patel y cols, en su serie de 1.500 PRR, su tasa de complicaciones fue del 5,08\% (Clavien I, $2.24 \%$, Clavien II 1,8\%, Clavien IIIa 0,08\%, Clavien IIIb 0,48\% y Clavien IVa 0,40\%), con un sólo caso que requirió de conversión debido a un mal funcionamiento del Robot ${ }^{12}$. En nuestra serie la tasa de complicaciones postoperatoria fue del $8 \%$ y todas clasificadas como Clavien 1.

La presencia de márgenes quirúrgicos positivos es un factor predictor independiente de recidiva bioquímica, por lo tanto, la evaluación de los mismos son fundamentales a la hora de evaluar los resultados oncológicos cualquiera que sea el abordaje quirúrgico. La tasa media de márgenes positivos publicada en PRR es de 15,2\% (9,3 a 33\%). Diferentes publicaciones han demostrado que la experiencia del cirujano hace que los márgenes positivos sean menores cuanto mayor número de casos se ha realizado. Atug y cols, evaluaron su serie de 140 PRR realizadas por el mismo equipo quirúrgico y los dividieron en 3 grupos ${ }^{16}$. La tasa de márgenes positivos para el primer grupo fue de $45,4 \%, 21,2 \%$ para el segundo y $11,7 \%$ para el tercero. Demuestran que a mayor experiencia del cirujano, menor es la tasa de márgenes positivos $(\mathrm{p}=0,005)$. La tasa de márgenes positivos en nuestra serie también se vio relacionada con la experiencia del cirujano debido que, para el grupo 1 la tasa fue del $20 \%$ y para el grupo 2 del $12 \%$.

Distintas series hacen referencia a los resultados sobre la continencia urinaria con diferentes definiciones. Al definir continente a aquel paciente que no requiere paños absorbentes o utiliza al menos uno por seguridad, Coelho y cols, publicaron una tasa de continencia de 13,1-38\%, 23-82,5\%, $47-93 \%, 54-97,1 \%$ y $70-97 \%$ a 1,3, 6 y 12 meses de seguimiento, respectivamente ${ }^{15}$. Al evaluar la continencia inmediata luego del retiro de sonda vesical, la tasa promedio publicada en la literatura fue del 25,7\%. Pocos estudios han comparado los 3 diferentes abordajes de la PR (abierta, laparoscópica y robótica). Tewary y cols, reportaron un estudio no randomizado en el cual compararon 100 PR abiertas vs 200 PR robóticas ${ }^{17}$. Los pacientes con el abordaje robótico tuvieron una recuperación de la continencia más rápido (44 vs 160 días; p < 0,05). Rocco 
y cols, reportaron también un mejor resultado en continencia comparado con la cirugía abierta ${ }^{18}$. Sin embargo, Krambeck y cols, demostraron una tasa de continencia similar entre el abordaje robótico y el abierto pero al año de seguimiento (91,8 vs 93,7\% respectivamente, $\mathrm{p}=0,344)^{19}$.

Finalmente, probablemente el parámetro postoperatorio más difícil de evaluar sea la potencia sexual debido a que múltiples factores actúan sobre ésta (edad, comorbilidad, calidad de preservación de bandeletas neurovasculares, etc.) así como la falta de estandarización en su evaluación. En la revisión publicada por Coelho y cols, la tasa media de potencia sexual a los 3,6, 12 y más de 18 meses, fue de $38,4 \%, 61,1 \%, 71,2 \%$ y $94 \%$, respectivamente ${ }^{15}$. Al comparar el abordaje robótico con la cirugía abierta, Tewari y Rocco, comunican mayores tasas de potencia para el abordaje robótico a los 3, 6 y 12 meses (robótica vs abierta: $31 \%$ vs $18 \%$ a los 3 meses; $43 \%$ vs $31 \%$ a los 6 meses y $61 \%$ vs $41 \%$ a los 12 meses; $\mathrm{p}=0,006$; 0,045 y 0,003, respectivamente) $)^{17,18}$.

Los resultados obtenidos en nuestra serie inicial se asemejan a aquellos centros de alto volumen quirúrgico. Los resultados están directamente relacionados con el mayor número de casos operados, determinando un descenso en los tiempos quirúrgicos, menor sangrado intraoperatorio y, sobre todo, una disminución en la tasa de márgenes positivos, de suma importancia como predictor de recidiva bioquímica, recurrencia local y posterior diseminación a distancia. Sin embargo, ¿cuántos procedimientos deben ser realizados para disminuir dichos parámetros determinando la curva de aprendizaje? En esta publicación demostramos estadísticamente que la curva de aprendizaje es francamente menor comparado con la laparoscópica, siendo de 40 procedimientos para la PRR. Recordamos que era de 100 para la PR laparoscópica, tal como fue demostrado por Castillo y cols ${ }^{20,21}$.

Como conclusión, el abordaje robótico de la PR proporcionaría una recuperación de la continencia precozmente en comparación con los otros abordajes $\mathrm{y}$, como demostramos estadísticamente, la curva de aprendizaje es menor comparado con el abordaje laparoscópico. La era de la cirugía robótica comenzó hace aproximadamente 10 años y en la actualidad ha desplazado a la prostatectomía radical abierta en múltiples centros. Sin embargo, es necesario estudios prospectivos y randomizados que comparen ambas técnicas quirúrgicas.

Si bien no es un estudio comparativo, nuestros resultados se asemejan a lo publicado. Necesitamos mayor tiempo de seguimiento para evaluar los resultados oncológicos y funcionales. Además, la experiencia del cirujano, directamente relacionada con el número de casos operados, hace a la PRR una técnica segura en relación a los parámetros perioperatorios y márgenes quirúrgicos.

\section{Referencias}

1. Jemal A, Siegel R, Ward E, Hao Y, Xu J, Murray T, et al. Cancer statistics, 2008. CA Cancer J Clin. 2008;58:71-96.

2. Hayat HJ, Howlander N, Reichman ME, Edwards BK. Cancer statistics, trends and multiple primary cancer analyses from the Surveillance, Epidemiology and End Results (SEER) Program Oncologist. 2007;12:20-37.

3. Pow-Sang M, Destefano V, Astigueta JC, Castillo O, Gaona JL, Santaella F, y cols. Cáncer de próstata en Latinoamérica. Actas Urol Esp. 2009;33:1057-61.

4. Guía clínica: Cáncer de próstata en mayores de 15 años. Junio 2009. Disponible en http://www.minsal.cl (Consultado el 15 de febrero de 2011).

5. Bill-Axelson A, Holmberg L, Ruutu M, Häggman M, Andersson SO, Bratell S, et al. Radical prostatectomy versus watchful waiting in early prostate cancer. N Engl J Med. 2005;352:1977.

6. Binder J, Kramer W. Robotically-assisted laparoscopic radical prostatectomy. BJU Int. 2001;87:408-10.

7. Intuitive Surgical Investor Presentation. Disponible en http://investor.intuitivesurgical.com/phoenix. zhtml?c=122359\&p=irol-IRHome (Consultado el 15 de febrero de 2011).

8. Orvieto MA, Patel VR. Evolution of Robot-assisted radical prostatectomy. Scand J Surg. 2009;98 76-88.

9. Rocco F, Gadda F, Acquati P, Carmignani L, Favini P, dell'Orto P, et al. Personal research: reconstruction of the urethral striated sphincter. Arch Ital Urol Androl. 2001;73:127-37.

10. Rosen RC, Riley A, Wagner G, Osterloh IH, Kirkpatrick $\mathrm{J}$, Mishra A. The international index of erectile function (IIEF): a multidimensional scale for assessment of erectile dysfunction. Urology 1997;49:822-30.

11. Dindo D, Demartines N, Clavien PA. Classification of surgical complications: a new proposal with evaluation in a cohort of 6336 patients and results of a survey. Ann Surg. 2004;240:205-13.

12. Patel VR, Palmer KJ, Coughlin G, Samavedi S. Robotassisted laparoscopic radical prostatectomy: perioperative outcomes of 1500 cases. J Endourol. 2008;22: 2299-305.

13. Zorn KC, Gofrit ON, Steinberg GP, Taxy JB, Zagaja GP, Shalhav AL. Planned nerve preservation to reduce positive surgical margins during robot-assisted laparoscopic radical prostatectomy. J Endourol. 2008;22:1303-9.

14. Badani KK, Kaul S, Menon M. Evolution of robotic radical prostatectomy: assessment after 2766 procedures. Cancer 2007;110:1951-8.

15. Coelho RF, Chauhan S, Palmer KJ, Rocco B, Patel MB, Patel VR. Robotic-assisted radical prostatectomy: a review of current outcomes. BJU Int. 2009;104:1428-35. 
16. Atug F, Castle EP, Srivastav SK, Burgess SV, Thomas $\mathrm{R}$, Davis R. Positive surgical margins in robotic-assisted radical prostatectomy: impact of learning curve on oncologic outcomes. Eur Urol. 2006;49:866-71.

17. Tewari A, Srivasatava A, Menon M, Members of the VIP Team. A prospective comparison of radical retropubic and robot-assisted prostatectomy: experience in one institution. BJU Int. 2003; 92:205-10.

18. Rocco B, Matei DV, Melegari S, Ospina JC, Mazzoleni F, Errico G, et al. Robotic vs open prostatectomy in a laparoscopically naive centre: a matchedpair analysis. BJU Int. 2009;103:448-53.
19. Krambeck AE, DiMarco DS, Rangel LJ, Bergstralh EJ, Myers RP, Blute ML, et al. Radical prostatectomy for prostatic adenocarcinoma: a matched comparison of open retropubic and robot-assisted techniques. BJU Int. 2008;103: 448-53.

20. Castillo O, Díaz M, Vidal I, Fonerón A, Sánchez-Salas R, Vitagliano G. Prostatectomía radical laparoscópica extraperitoneal: Resultados de una curva de aprendizaje después de 310 casos. Rev. Chil Cir. 2009;61:52-8.

21. Castillo OA, Vidal-Mora I, Sánchez-Salas R. Prostatectomía radical laparoscópica. Rev Chil Cir 2011;63:21722. 